\title{
Baroque
}

\section{BAROQUE 09-10|1980}

Méthodologie

\section{Préambule}

Félix-Marcel Castan

\section{(2) OpenEdition}

\section{Journals}

Édition électronique

URL : http://journals.openedition.org/baroque/519

DOI : 10.4000/baroque.519

ISSN : 2261-639X

\section{Éditeur :}

Centre de recherches historiques - EHESS, Éditions Cocagne

\section{Édition imprimée}

Date de publication : 1 mai 1980

ISSN : 0067-4222

\section{Référence électronique}

Félix-Marcel Castan, «Préambule», Baroque [En ligne], 09-10 | 1980, mis en ligne le 15 mai 2013, consulté le 20 avril 2019. URL : http://journals.openedition.org/baroque/519 ; DOI : 10.4000/ baroque. 519

Ce document a été généré automatiquement le 20 avril 2019

(c) Tous droits réservés 


\title{
Préambule
}

\author{
Félix-Marcel Castan
}

\section{Intervention}

1 La session de 1976 restera étroitement tributaire des résultats de la session de 1974 : la critique du concept de Baroque, ou plutôt de son statut, doit s'entendre comme préalable à une réflexion éminemment constructive. La procédure proposée dérive de la notion de dépassement.

2 Dépassement du monologue des spécialistes. - Nous avons entendu dès l'origine, en 1963, ce que des spécialistes disaient à des spécialistes d'autres disciplines : les thèmes des débats visaient à une signification ordonnée. En 1974 surtout, l'intensité de l'échange et son caractère essentiellement réflexif a fait concevoir un au-delà de l'interdisciplinarité, où pourtant on n'entendait que la parole du concret disciplinaire, dont l'écoute se trouvait renouvelée: ce lieu d'écoute actualise ce qu'on nomme communément civilisation. Il en justifie le terme.

Dépassement du moment de la parole. - Jamais ces Journées ne se sont placées dans le cadre d'une typologie constituée du Baroque : au contraire elles ont d'emblée ouvert leurs débats à toutes les doctrines possibles, sans discrimination. Ce que chacun dit contribue à une approche du moment culturel, défini par sa globalité. Les discours doivent à leur convergence et à leur inachèvement leur force d'intégration et d'orientation. On ne saurait autrement ériger le qualificatif baroque en substantif.

Dépassement des critères, des thématiques, des formes, des figures, des structures. - Le colloque ne mobilise pas seulement les systèmes doctrinaux, il fait aussi bouger les concepts qui tendraient, sinon, à occulter le point omega (ou point aveugle ?) du Baroque. Ceux-ci s'établissent dans une relativité mutuelle, mettant en évidence aussi bien leurs carences que leur positivité, au service d'une vérité de position, excluant toute idée de modèle épistémologique. 
Dépassement des analyses et des esquisses synthétiques. - Le colloque concret n'est que la figure ou le reflet du grand colloque invisible, et néanmoins constant et universel, de la recherche. Il ignore la clôture: simple temps de recul par rapport au flux d'une recherche illimitée, il vise à renforcer la compétition des acquis théoriques, à développer les tensions, voire les contradictions.

6 Dépassement des démarcations géographiques et historiques. - Par-delà les frontières spatio-temporelles des formations sociales, la science tend à instaurer ses propres structures de connaissance, tour à tour poreuses et étanches, selon le point de vue interne ou externe d'où on les considère. Le Baroque ne se présente pas comme un Empire, mais comme un laboratoire. La pensée humaine s'y saisit dans la fréquentation des actes accomplis de l'humanité, qu'elle démêle par différenciation progressive et par la mise en activité d'autant de laboratoires spécifiques qu'elle décèle d'ensembles de même niveau ou de pôles distincts.

Dépassement de la continuité des courants internationaux. - À partir de la notion de Laboratoire, où se confrontent les phénomènes et les idées qu'on s'en fait, le principe de continuité objective perd de son intérêt. L'unité se construit par degrés au cours de la connaissance, selon un processus de sédimentation et en quelque sorte de nidation. On pourrait, pour désigner les sciences de la culture, forger le terme de science de composition, par analogie avec les rôles de composition au théâtre, ou de science au deuxième degré, afin de les distinguer des sciences au premier degré (sciences de la nature et sciences dites humaines), ou encore de science des surdéterminations collectives.

8 Le thème retenu a paru répondre aux conditions requises pour éviter le blocage des discussions. Il sort des débats de 1974, dans la mesure où ceux-ci ont nourri, de l'inquiétude du négatif, un germe de positivité. On peut croire qu'une civilisation se caractérise plus par ce qu'elle construit que par ce qu'elle détruit, et qu'il n'est pas de rupture qui ne se révèle à la fois négative et positive. Certains moyens d'expression semblent nés au cœur de l'aventure baroque, générés par son mouvement : l'opéra italien, la comedia espagnole, l'essai de Montaigne, l'algèbre de Viète, les équations de Képler, par exemple. Plus généralement, les divers modes d'expression semblent évoluer et s'ouvrir de nouveaux chemins.

9 Toute gestation culturelle s'accompagne sans doute d'une promotion du Logos. Il reste, dans le cas présent, à apprécier la nature et l'importance de cette promotion. S'agit-il d'un véritable avènement épistémologique, d'une libération phénoménologique?

\section{Discussion}

10 - CASTAN : Nous sommes pour la deuxième fois réunis pour parler de méthodologie. La proposition de thème est sujette à débat, sur le principe même. En 1974, nous avions pensé que la définition du Baroque devait être rejetée par hypothèse, que le concept ne pouvait être remplacé par d'autres concepts. Cette année, dans la mesure où nous serons tentés de dire B «Le Baroque, c'est...", nous ne serons pas dans la ligne de notre recherche. La formule "Baroque, promotion du logos» ne doit pas être entendue comme: le Baroque, c'est la promotion du logos. Toutes les époques de civilisation connaissent une promotion du logos. Comment, dans le siècle considéré, la raison humaine a-t-elle progressé, s'est-elle saisie de ses pouvoirs? « Nous allons considérer le 
Baroque en tant que promotion du logos, nous demander de quelle manière le logos a progressé à cette époque, comme il a progressé aux autres époques.

11 En 1974, ici même, un historien des sciences, le Père Costabel, dit à peu près :

À cette époque-là, toutes les croyances sont dans une situation d'effondrement. On ne sait plus sur quoi il faut s'appuyer. Les essences de la réalité auxquelles on croyait antérieurement, fondées sur des croyances religieuses ou non, sont en déroute.

12 Que reste-t-il dans ce malaise de civilisation, qui est fondamental ? Une capacité pour l'esprit de raisonner, de formuler des lois : c'est la bouée au milieu de cette mer démontée. La faculté de logos. Événement qui oriente l'avenir de la civilisation. Dans le domaine littéraire, certains ne croient plus qu'aux capacités du langage de se réaliser par ses propres forces, sans s'appuyer sur une philosophie antérieure. Montaigne fait confiance à la seule écriture, pour retrouver un mode d'appréhension du réel, un mode d'existence dans le monde. Voilà à partir de quelle expérience notre proposition a été formulée ${ }^{1}$. C'était aussi à quoi nous invitait en 1974 l'intervention de Fernand Drijkoningen.

Quant à son interprétation, deux aspects :

- quel rôle le logos est-il appelé à jouer, un rôle plus profond, plus essentiel qu'il n'avait joué jusque-là dans le fonctionnement de la civilisation?

- comment les formes mêmes de la raison se sont-elles multipliées, comment les pouvoirs de l'homme sur la réalité se sont-ils accrus?

Problème de synthèse, qui pénètre toutes les activités, et permet d'éclairer en profondeur tous les secteurs disciplinaires. Ce sont des spécialistes de la littérature, de l'histoire de l'Art, des Sciences, etc. qui parlent du Baroque. On ne voit pas de spécialistes du Baroque détachés de l'activité sectorielle : ils centrent seulement alors leur réflexion à un niveau où s'élabore une synthèse de civilisation, où véritablement le problème de la science des civilisations est posé. Y aura-t-il un jour, dans les universités, des chaires du Baroque ? Le discours collectif commence...

15 - MARTINON: Dans le texte introductif, une petite phrase me semble, méthodologiquement mais aussi d'un point de vue épistémologique, tout à fait pertinente: «Le Baroque ne se présente pas comme un empire, mais comme un laboratoire ». Cette phrase pose et situe la question fondamentale de l'éclatement, de la volatilisation du concept de baroque tel qu'il avait été utilisé dans les premières sessions $\mathrm{du}$ colloque qui avait pour sujet le statut de ce concept. Il semblerait que le mot baroque soit à la fois présent et insaisissable. D'un côté nous sommes confrontés à la présence de son empirie - je ne dis pas empire - qui se matérialise en une multiplicité presque hétéroclites de matériaux et de documents (monuments et architectures, sculptures, jardins, textes littéraires et religieux, comportement- et pratiques de différents groupes sociaux, etc.). D'un autre côté, nous sommes confrontés à l'insaisissable : le baroque est présent en empirie mais il est insaisissable en théorie. Comment élaborer une synthèse à partir de matériaux et des documents que nous répertorions et interprétons ? Comment construire une science du baroque ? Il y a là du constitué, mais on ne sait pas bien ce que peut être le constituable et pourtant le secteur empirique déterminant l'extension du baroque est déjà, institutionnellement, constitué par la présence, oh combien insistante ! de nos différentes disciplines universitaires. 
16 Nous avions commencé à parler en 1974 du baroque comme fait de civilisation, comme un fait social total, nous avions essayé de découvrir une cohérence - oserons-nous dire structurale - de toutes les facettes de l'événementiel baroque mais, évidemment, il s'agissait d'un vœu et d'un projet à long terme, car il est nécessaire que la multiplication des travaux empiriques soit accompagnée par une réflexion sur le découpage de l'objet d'étude. Ce que je viens de dire est bien naïf puisque tout découpage théorique présuppose un objet d'étude déjà spécifié culturellement selon les modalités des classifications mises en œuvre par les spécialités d'un champ d'étude qui, dans le même mouvement réflexif est à la fois découpé et découpant. Il faudrait relire Max Weber à ce propos. Pourtant la question concernant le statut du baroque reste entière. D'une part, comment découper un champ de réflexion et d'études qui serait le lieu d'un discours à prétention scientifique et qui s'imposerait comme fondateur du baroque? Voici la question importante. D'autre part, ne serions-nous pas, en tant que spécialistes possédant ou croyant posséder une expertise spécifique, en train de cheminer sur des routes parallèles, chacun étant confiné dans sa spécialité sans pouvoir en sortir? Vous connaissez, à ce propos, le passage dans L'Homme sans qualité de Musil :

[...] l'homme commence à réfléchir sur la vie dans son ensemble, mais plus il réfléchit avec précision, plus son domaine se rétrécit. Quand il a atteint la maturité, tu as devant toi un homme qui est si ferré sur un certain millimètre carré qu'il n'y a pas dans le monde entier deux douzaines d'hommes aussi ferrés dans ce domaine. Il voit fort bien que les autres, moins ferrés que lui, ne disent que des bêtises sur ses affaires, et pourtant il ne peut bouger, parce que c'est lui, s'il quitte sa place, ne fûtce que d'un micromillimètre, qui en dira à son tour.

Quelque chose était acquis au colloque de 1974: nous avions laissé les couteaux de la spécialité au vestiaire. Il ne s'agissait plus de débats entre spécialistes murés dans leur spécification autonomisante, au contraire, une réflexion unifiante, sans être bavarde, traversait les spécialistes. Souvenons-nous de cet aphorisme de Lichtenberg : « en battant la campagne sans méthode, en s'abandonnant sans méthode aux vagabondages de la fantaisie, il n'est pas rare qu'on lève le gibier que la philosophie méthodique pourra utiliser dans son ménage bien en ordre ». Nous étions en 1974 à la croisée des chemins, entre Musil et Lichtenberg et une recherche s'élaborai t: celle de l'extension du mot baroque : les limites sont-elles historiques et/ou géographiques ?... peut-être ici faut-il se souvenir de l'élaboration d'une science comme celle de l'histoire comparative des religions... y aurait-il des œuvres, des faits de société que nous pourrions comparer avec pertinence et que l'on pourrait subsumer sous le mot de baroque sans pour autant être taxé d'essentialisme ? Voici la situation du colloque en 1974 : il s'agissait d'une situation d'effondrement du concept de baroque mais aussi d'une situation de conversion, de retournement sur soi-même et sur ses propres pratiques d'investigations scientifiques.

18 - CASTAN : Est-ce ainsi que tout le mande a ressenti les journées de 1974 ?

19 - AUZIAS : Aujourd'hui, ici, il n'y a plus de communications, parce qu'il y a de la communication. Autrefois, des spécialistes apportaient les pierres d'une mosaïque qui ne constituaient jamais un dessin. Consolons-nous par la qualité de l'écoute. Les spécialistes sont engagés dans ce jeu de miroirs par lequel ils se regardent eux-mêmes, et ils ne font que dédoubler à l'infini dans un narcissisme éternel leur propre crainte devant l'objet.

Il faut beaucoup se promener. Je suis allé au Portugal, en Italie, au Pérou : pas pour des motifs esthétiques, mais pour travailler à la réforme agraire... Pour voir aussi dans quel contexte passé et présent le Baroquisme s'était développé et continue encore. Les 
spécialistes ne sont d'aucun secours, car ils sont toujours tentés de faire une superposition mécaniste d'une œuvre et du discours social qui la sous-tend. Simple jeu. La bonne méthode repose sur un refus fondamental de tout mécanisme. Pour tout dire, je crois depuis deux ans que l'unité c'est l'anthropologie, la sociologie et l'économie qui nous la révèlent, dans l'histoire. L'extraordinaire déploiement du monde à cette époquelà est essentiellement réglé par une réappropriation du monde, qui n'est pas celle des gens qui regardent, mais celle des gens qui donnent à voir. Cette expansion et cette maîtrise du monde sont immédiatement confisquées, comme le montre le système du travail en Amérique du Sud : pour des gens qui n'en sont pas même à l'étape du salariat, c'est le système de la plus-value totale... Vous excuserez mes références marxistes aux systèmes de production. C'est peut-être dans la direction de cette étude du système général du travail que nous trouverons l'unité du monde baroque. Il reste à savoir comment sur cette unité du monde se sont élaborées des formes. Comment ces formes, qui sont données comme auxiliaires d'une répression générale, deviennent en réalité des formes de libération. C'est ce qui me paraît être le mystère du Baroque.

21 - LAFAY: Je suis là en sceptique. J'ai perdu la foi baroque. J'étais des premiers dans votre travail, et je ne peux plus employer le mot Baroque. Je constate qu'il est employé par d'autres, problème pour moi. Martinon a bien expliqué comment la dernière fois nous n'étions pas parvenus à constituer le mot Baroque. Vous avez parlé de ce parallélisme des spécialités qui ne communiquent pas. Vous saisissez la difficulté en synchronisme. Elle est vraie. Personnellement, je verrais une autre difficulté en diachronie : je me demande si on n'emploie pas le mot pour cacher une nébuleuse, base de télescopages de réalités historiques diverses.

Deuxième réflexion : perplexité devant le thème « promotion du logos ». J'ai envie de dire exactement le contraire : déroute. éclatement du logos, pulvérisation, anéantissement. Christeva ou J.-M. Benoist font des analyses qui pourraient être des réhabilitations du Baroque par critique du logos platonicien: celui-ci serait une symbolisation réductrice par rapport à la réalité. La réalité est remplacée par le mot, et ensuite tout se passe au niveau du logos et non de la réalité.

Mais vous parlez d'une capacité du langage de se réaliser lui-même par ses propres forces. Ce serait un retour à la réalité au niveau du mot, qui n'est plus le substitut de la chose. Pour Auzias ce serait une sorte de réapparition du monde au niveau du langage ultrasymbolisé (substitut abstrait). Je pars sans idées. Je suis venu pour écouter, pour être remis en cause. Je commence par des paroles confuses. Notre maître commun Jean Lacroix nous disait : il ne faut pas penser avec des idées claires.

24 - Précisément pour la période dite maniériste, nous sommes dans un logos de type platonicien : vous ne pouvez pas étudier la pointe, le concetto sans passer par cette idée. - Pouvez-vous préciser les positions de M. Benoist là-dessus.

26 - LAFAY : Cette philosophie du logos apparaît chez les Grecs comme fondement de la civilisation occidentale : à la réalité est substituée un logos. Je verrais le Baroque comme une réappropriation du réel, échappant à cet univers de substitution, l'univers du logos. C'est de cet univers que Christeva et Bénoist veulent sortir pour retrouver la perspective freudienne de destruction du logos, les traces pulsionnelles de vie. Déroute du logos. Revanche de la réalité, qui se manifesterait de manière différente selon les moments, et il faudrait reprendre les analyses marxistes pour les différents moments. 
27 - WAGNER : Je suis dans l'ignorance totale des travaux de Benoist et de Christeva. Vous m'expliquez que le mot est substitué à la chose, je ne vous suis pas. Il m'apparaîtrait plutôt que ce devrait être l'image. Mais si c'est l'image, nous ne sommes plus dans le langage. Nous sommes dans une symbolique, évacuant le contenu. Qu'on puisse dire que le mot s'est substitué à la chose ne me semble pas une position strictement platonicienne.

28 - Marie-France SALQUES : Dans la symbolique, vous avez dualité : l'image platonicienne est chargée de contenu, puisqu'elle est chargée de l'idée. C'est bien le drame !

29 - AUZIAS : Tout un courant actuel pense qu'avant de parler je ne savais pas ce que j'allais dire ; quand j'ai fini, je ne sais pas ce que j'ai dit. Entre-temps j'ai peut-être travaillé sur la matière. Le langage est sa propre réalité et l'idée du modèle préexistant est évacuée. Passage de la linguistique classique à la linguistique générative.

30 - WAGNER : La critique du modèle préexistant touche-t-elle la catégorie du Baroque? Dans le domaine qui est le mien, l'histoire de la philosophie, la catégorie en soi ne m'intéresse que parce qu'elle peut, sous certaines conditions, devenir opératoire, me permettre devant un texte d'intégrer le côté rapport au monde de toute philosophie. Je m'interroge : est-ce que la critique du logos platonicien dont vous parlez est une critique $\mathrm{du}$ logos platonicien renaissant?

31 Pour ce qui est de la période dite maniériste par certains, nous sommes en plein logos de type platonicien (concetto...).

32 - LAFAY : La pointe correspondrait-elle à une sorte de vie propre du signifiant, sans référent réel.

33 - WAGNER : Je ne le crois pas : on a affaire ici à la présence simultanée de deux contraires, de deux extrêmes.

34 - HALLYN: À l'époque baroque, me semble-t-il, la science dans son retour aux mathématiques est platonicienne. Mais la rhétorique est aristotélicienne, il n'y a pas d'idées préconçues, c'est à travers le signifiant que les idées se construisent. Opposition qui sous-tend toute l'époque : il y a à la fois construction et déconstruction du platonisme.

35 - WAGNER: Rhétorique purement aristotélicienne, je vous crois. En revanche, la, rhétorique de la Renaissance est incontestablement platonicienne (avec des règles aristotéliciennes), de même que la noétique. Ce qui reste à savoir c'est si derrière une rhétorique aristotélicienne ne se trouvent pas paradoxalement des visées platoniciennes (j'y reviendrai). Cette critique baroque du logos platonicien, est-ce la critique du logos de la Renaissance?

36 - HALLYN : Dans la rhétorique de la Renaissance, il y a en effet les deux, platonisme et aristotélisme - Mais non dans la rhétorique du Baroque.

37 - Hana TECHOVA : Il y a oscillation, hésitation entre les deux pôles, dans les textes mêmes. Dans un même pays, le rapport au logos, chez les écrivains protestants par exemple (Comenius) et chez les Jésuites n'est pas le même. Il faut les étudier à leur place, dans la géographie, on s'en rend compte quand on les traduit comme j'ai eu à le faire. Les images des Jésuites, images autonomes, ne se rattachaient à la réalité que d'une façon arbitraire, tandis que chez Coménius.les images étaient explicatives, transmettaient une idée, et sortaient d'une tradition antérieurement constituée, d'où la difficulté de traduction. Deux types d'images, deux aspects de l'expression baroque.

38 - Marie-France SALQUES : Je reviens en arrière. Je ne vois pas d'opposition entre ce qu'a dit Lafay du signifiant, du mot ou de l'image qui avait en quelque sorte évacué le contenu 
et survivait par ses propres forces, et d'autre part la pointe avec ses extrêmes saisis par l'ingenio. L'ingenio, en l'occurrence, a un rô1e très particulier, puisqu'il est censé être très exactement la même chose pour le créateur de la pointe et pour son lecteur (première ambiguïté éclairante): il est le substitut de l'ancien contenu du logos platonicien (ou même du contenu du néo-platonisme de la Renaissance). C'est un fait que dans la rhétorique et dans le langage baroques on peut analyser une tendance platonicienne et une tendance aristotélicienne. Quant à leurs places respectives, il me semble que le logos platonicien serait plutôt un point de départ qu'une visée. On l'utilise au départ, pour le nier.

39 Ceci grâce à un processus de renversement du logos dont je vais donner une image très simple, courante chez certains poètes marinistes. Qu'on pense à l'importance accordée à la lune en tant que reflet du soleil. Le soleil, dans le monde du maniérisme, a relativement peu d'importance: le symbolisme lunaire comme reflet, c'est la réhabilitation de la lumière humaine. Autre exemple : l'éclat des pierres précieuses, qui brillent par ellesmêmes dans l'obscurité. Non pas négation du logos platonicien, mais renversement qui, plus ou moins mêlé chez certains auteurs à un contexte chrétien, est à la limite du blasphème.

Pour résumer, le logos platonicien semble omniprésent dans la rhétorique baroque, comme point de départ commode, l'ingenio ayant évidemment un rôle considérable dans la négation par un renversement systématique. L'ingenio du poète qui crée et celui du lecteur qui lit avec la même capacité d'intelligence, sont dans une parfaite équivalence par rapport à un plan médian qui serait le langage. Il y aurait finalement deux références, cette lecture et cette création.

41 - AUZIAS: Si j'étais historien de la philosophie, j'ajouterais que le modèle de la rhétorique, c'est alors Sénèque. Ceux qui mettent en œuvre le logos, Lily en Angleterre, Gongora en Espagne, se réfèrent à Sénèque. J'entends dans votre discussion un écho des discussions actuelles sur la notion de modèle, en linguistique, en physique, etc. On est en pleine ambiguïté, dites-vous. C'est qu'il y a des œuvres, il y a des pratiques. Très bien ce que tu dis sur le concetto, Marie-France, c'est vrai. Mais qu'est-ce qui se passe en réalité ? Voilà des gens qui croient qu'ils font du Platon, du Sénèque, et qui font tout autre chose. Les explications théoriques nous renverront toujours à ce qui a été écrit sur, comme si c'était les universitaires qui faisaient le monde. Ce n'est pas eux! Ils sont dans un certain monde, et qu'est-ce qui se passe dans ce monde, pour qu'il y ait une telle confiance dans un discours qui même chez les Jésuites s'autonomise ? Pourquoi est-ce que ce discours est de plus en plus autonome? Lorsque vous discutez des images des Jésuites et de la mort, j'aimerais que vous me disiez de quoi il s'agit, parce que je suis très ignorant et je ne fais pas toujours ma prière avec les exercices d'Ignace de Loyola. J'aimerais savoir comment Ignace de Loyola se représente le monde.

-WAGNER: Cette méthode structuraliste que nous essayons d'employer pour comprendre ces thèmes, quant à moi, je ne suis pas du tout d'accord. Il ne s'agit pas d'établir une équivalence entre le modèle structuraliste et une idée de la Renaissance. Il s'agit au contraire, pour nous, de partir des ouvrages théoriques de la Renaissance, et il y en a (ils apparaissent d'ailleurs au début du XVII ${ }^{e}$ siècle et parfois très au-delà : Pelegrin 1638, Gracian 1606) : ils nous donnent une méthode pour aller aux textes. sont valables, parce que ce sont deux sommes qui contiennent pratiquement toutes les 
problématiques. Bien sûr, il n'y a pas dans les sciences opposition absolue à une prépondérance aristotélicienne. Mais Koyré, dans tous ses ouvrages montre l'apparition d'un nouveau platonisme dans les sciences.

44 - WAGNER : Tesauro, Pelegrini, Gracian, les «tractatistes ", sont très souvent des auteurs du XVII ${ }^{e}$. Certains les qualifient de théoriciens du maniérisme. Mon propos n'est pas de soulever le problème maniérisme/baroque, mais...

45 - Remarques diverses d'où émerge un consensus : quand Huygens établit son Cosmothéoros, déjà Newton perce en-dessous. Chaque fois que prend corps un discours cohérent, une réalité qui n'est pas cohérente le déborde. C'est à partir de là que la science devient possible, comme recherche toujours inachevée. Le début du XVII ${ }^{\mathrm{e}}$ siècle : moment où se constitue la science.

46 - AUZIAS : C'est pourquoi je vous dis : attention! Le discours de la répression fait tout rentrer dans le modèle, il modélise tout, comme si tout allait pouvoir y rentrer.

47 - Le modèle ne reste pas intact ! La répression est donc elle-même très relative.

\section{NOTES}

1. Pierre Costabel, regrettant de n'avoir pu participer à ce colloque, m'écrivait, le 22 octobre 1976 : «J'en suis d'autant plus navré après coup que, comme vous me le disiez, vous aviez fixé un thème que je vous avais moi-même suggéré. Vous m'aviez parfaitement bien compris... Du moins soyez assuré que le thème, fondamental, reste pour moi le moteur principal de mes recherches actuelles, et, par conséquent, je pense que nous aurons à en reparler ». 